\title{
Analytical Study of University Campuses' Walkability using Space Syntax Analysis: University of Duhok (UoD) as a Case Study
}

\author{
Jowan Bayez Khorsheed* and Wafaa Anwar Sulaiman Goriel
}

*College of Engineering, University of Duhok, Iraq

* Email: jowan@uod.ac; Corresponding Author.

\begin{abstract}
Human health is an essential issue for many decays; Walking is one of those human activities that showed optimistic results, "A healthy mind is in a healthy body". University as a small city as such consider healthy and should be environmentally friendly when it been designed responsively for sustainable criteria and human respects. Where Students, staff need for safety and comfortable work environment been satisfied. University of Duhok (UoD) as a campus have a lot of transportation problems, especially at break and peak hours. One of these main problems are car-oriented transport, lack of smart well-planned transport system. Thus, the research will be tackling those issues, by utilization the theory of Space syntax and using an analytical tool, depthmapX, Axial Analysis, to explore the potentials of the network system as an indicator of pedestrian satisfaction of walkability. The investigating of students' walkability projected through visual observation, streets graphic documentations, a digital simulation of possible pedestrian paths through graphical analysis of site elements and components. This research aimed to educate the community and stockholders on the possibility of promoting walkability.
\end{abstract}

Keywords: Walkability, Space Syntax, Sidewalks 


\section{INTRODUCTION}

For many decays, studying relationships between human behavior and built environment were ignored by the architects, urban designers, and planners. Walking as behavior have a social and morphological aspect. Social, is a term of health (mental and physical) and cost (economical). While, morphologically, walking for any community needs, where the walking space, space accessibility, and cost (time and distance) are affordable, etc. Also, it is essential to consider safety, space coherence where the space navigation from-to destination is varied from one to another. Thus, this research developed a framework that's aided by visual, graphical, and syntax analysis, urban design and planning theories and focusing on walkability. Where the theories and methods have been tested in the case study of the University of Duhok in Duhok, Iraq.

\section{THEORETICAL FRAMEWORK}

\section{WALKABILITY}

Walkability is an indicator of a well-designed, and safe site, where sidewalk and services are well prepared and designed. The walkability of the site is a strategy for a healthier, safer, convenient, and active University campus. It's not only important for reducing congestion, cleaner environment, social and recreational value rather it promotes health physically and mentally (Southworth, 2005). People's behavior can be shaped when architects, urban designers, and planners worked smartly on the design of the built environment (Keat, Yaacob, \& Hashim, 2016). Walking path should be friendly, safe, secure, coherence, and attractive, Pedestrian safety is related to the relationship between pedestrian and built environment around (Fruin, 1992). Streets network is the backbone of cities, to be viable and active as well as to the university as a small city. Pedestrian spaces consist of street furniture, sidewalks, and lighting. Sidewalks can be convenient if it was clear from obstacles (e.g. fire hydrant and trash bins, etc.). 


\section{SMART CITIES AND WALKABILITY}

'University' is a center for science, education, accommodation, culture, and recreation facilities that contribute to the city weal fear and development (Bolshakov, 2019). Integrating the concept of smart City (Jones, Marshall, \& Boujenko, 2008), addressed binaries In Urban design and planning. Where the same street once can be used as a channel of movement (e.g. used by vehicles, public transportation, cyclist and pedestrian) simultaneously, it can be used by people for standing, sitting, sightseeing, or even for a public and social events. The concept is about utilizing the same geographical occasion to be a channel of movement as well as space for activities and this called a smart design and smart cities. Any university is having an external and internal connection, while external is linking the campus with the urban transport and the city as a whole, the internal is gluing the campus itself as one unit (Bolshakov, 2019).

\section{SPACE SYNTAX THEORY}

According to space syntax theory, previous definitions, "it is a method used to describe and analysis the relationships between public spaces and buildings". Normally, architects refer to those relationships as "Design Layout.”. Klarqvist (2015) demonstrated that Hillier and his colleagues have applied to urban studies how to deal with the urban spaces: First: The way urban space, Second: The techniques used to analyze cities, Third: set these techniques to observe how these space networks relate to functional patterns such as movement and fourth: spatial syntax has enabled a set of theories about how relate to the social, economic and cognitive factors that shape them (Van Nes, 2014). Space syntax indicators and According to Hillier's study, an interactive network for both movements to and through spaces is an essential requirement, where sets of trip origin and destination, or to-from node are offered and route through the predefined location is planned since routes leading to those locations will be more likely to be placed (Hillier \& Iida, 2005). 


\section{RESEARCH PROBLEM, AIMS, SIGNIFICANCE}

From the previous studies, it concluded that people are more willing to walk for smaller blocks, pedestrian-friendly, shorter walking journeys in safer sidewalks. The Research Question and Problem concerns with: "Why there is no walking in UoD? What do we need to create a walkable campus?". The creation of a walkable Campus means implementing walkability improvement for the whole university and encouraging the students and staff to engage, walk, and create a walkable area. The research presenting an analytical study for UoD Master Plan to explore the campus potentials. To achieve the study's objectives it needed to: 1- Identify the walkability Areas within the University of Duhok Campus.2- Using the Syntactical Analysis that shows high-Low connected streets. 3- Address the Pros and cons of UoD sidewalks, quality, and safety.

\section{RESEARCH METHODOLOGY, METHODS}

Universities is a small urban city, where many activities are taking place such as Walkability. it was essential to answer the question, "Why there is no walking in the University of Duhok (UoD), see Table 1. A selected methodology for the implementation of "University campuses walkability" is designed based on sets of indicators; urban morphology which is presented by (walkability network, building layout, and entrances location) and urban walkability indicators. While campus walkability is representing individual choice for walkability effected by design standards and sidewalks infrastructure availability. The research methodology aiming to link morphological indicators with walkability, to get a quantifiable human behavior (Figure 1). 
Table 1. UoD Campus Walking Problems

\begin{tabular}{|l|l|}
\hline Why there is no walking at the University of Duhok? \\
\hline UoD's Campus Problems & \multirow{2}{*}{ Method used } \\
\hline No pedestrian paths & Graphic Analysis, Street Sections, Documentations \\
\hline Designed Sidewalks infrastructure & \multirow{2}{*}{ Space Syntax Analysis (depthmapX) } \\
\hline Green paths, sitting Areas & \\
\hline $\begin{array}{l}\text { Public areas, squares } \\
\text { Pedestrian accessibilities, Connectivity to service points (Restaurant, }\end{array}$ library, college building entrances, Car parking, central libraries) &
\end{tabular}

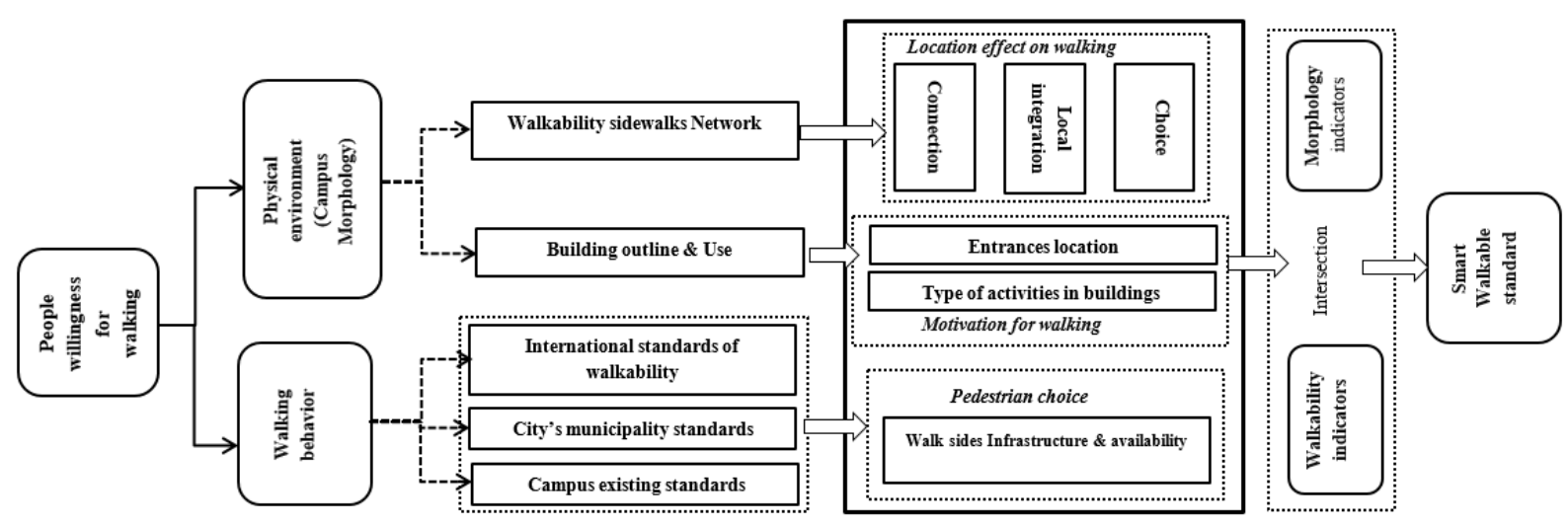

Figure 1 Conceptual framework

The method was carried out by the application of graphical and Syntactical Analysis computation through the "depthmapX" software. Where ground-controlled data is used for investigating the real state of UoD sidewalk and compared with the resulted index from space syntax (Connectivity, Integration (Local and Global), and choice (Local and Global)) showing the effect of street connectivity, integration and sidewalk infrastructure on individual choice.

\section{GRAPHICAL ANALYSIS}

It is one of the architect's languages (Brusaporci, 2015) that been used for expressing another language that used to present ideas and solutions for specific design. It can be coded drawing which stands for fundamentals in a built environment (e.g. structural, functional solution, or even a 3D projection)(Brusaporci, 2018). The graphic analysis is one of the techniques that explain the reality in an engineering drawing environment. 


\section{SPACE SYNTAX TOOL (in depthmapX)}

"Space syntax analyses examine the relationships between components of space; each analysis starts with make a graph of these components, (Turner, 2004). Where it can be applied using a computer through depthmapX. (Turner, 2004). DepthmapX is a programmed research tool, as a computer program to perform a visibility analysis, axial analysis and segment analysis of architectural and urban systems. It is based on the concept of developing the space syntax". (Turner, 2004), (Hillier, Hanson, \& Peponis, 1984).

\section{FILED WORK AND DATA COMPILATION UNIVERSITY OF DUHOK: STUDY LOCATION}

University of Duhok (UoD) (Figure 2) is located in northern Iraq in the Kurdistan region. UoD is in the east of the Duhok city, on the main international highway road linking Iraq to Turkey and Syria. The University of Duhok established in 1992 with two colleges extended to eighteen todays. Which makes it promising as an educational and research Centre. UoD is built on hilly land with a designed master plan in 2005 , the arrangement of the master plan is based on one main ring road connecting the main educational sectors and its service buildings.

Data usedTo carry out this research, certain types of data collection and compiling carried out.

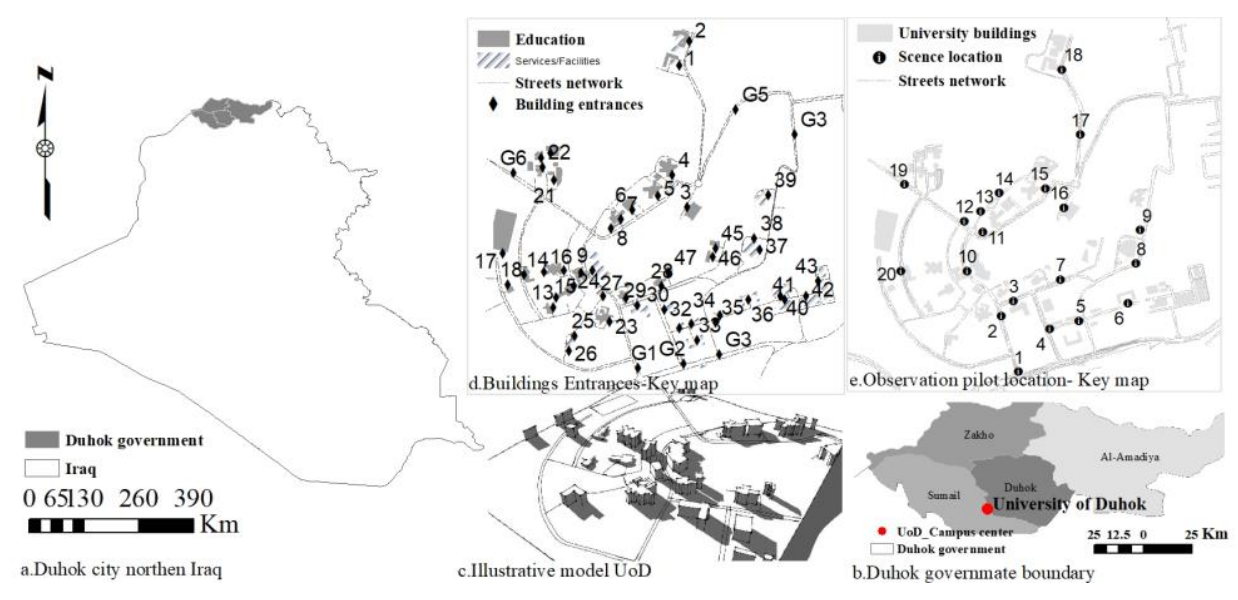

Table (2) 


\section{RESULT DISCUSSION}

The research built its analysis based on two main components, first the graphical analysis and second Space syntax analysis: UoD Street, sidewalks compare to local and international standards, any urban planner or a designer should follow a certain type of standards while designing and developing a city design. These standards are either depending on city local standards or based on selection of an international approved standard (e.g. European cities

Figure 2 University of Duhok: Study Location

standards, United States standards, etc.). Thus the researcher saw that it will be logical to compare the reality of UoD sidewalks with, Duhok city municipality (Abdulsamad, Mohammed, \& Abdullah, 2019) and international standards (San Diego, 2017), (San Francisco, 2020) see Table 3. From Figure 6.a. UoD sidewalks show big variation compare to Duhok municipality standards (Table 4), Duhok municipality standards are specified to 1.5 meters for $7-\mathrm{m}$ street width whereas UoD campus street is recorded from 4-8 for $6 \mathrm{~m}$ - 7-m street width. While comparing it to the international standards we find it higher than mean of 3.7 m-4.6 m for mixeduse streets see Figure 6. b. In general, UoD standards seem to be higher than local and international standards. Where, a further step is necessary to overcome the subjective judgment of whether our walk side's infrastructure is sufficient or not, a space syntax analysis has been carried out for the next phase.

Table 2 UoD Campus: Research Database

\begin{tabular}{|l|l|l|l|l|}
\hline Source & Tools & Description & Reference map & Figure \\
\hline $\begin{array}{l}\text { Geometrical map } \\
\text { for campus }\end{array}$ & $\begin{array}{l}\text { CadMapper- Online data } \\
\text { acquisition }\end{array}$ & $\begin{array}{l}\text { Centerlines of roads } \\
\text { campus of UoD }\end{array}$ & $\begin{array}{l}\text { Dwg/ AutoCAD files, Sketchup (3D } \\
\text { Model for illustration) }\end{array}$ & Figure 2 \\
\hline $\begin{array}{l}\text { Spatial Analysis } \\
\text { for space syntax }\end{array}$ & depthmapX software & $\begin{array}{l}\text { Outline of the whole } \\
\text { campus of UoD }\end{array}$ & Dwg/ Dxf. AutoCAD files & Figure 6 \\
\hline & $\begin{array}{l}\text { Real-life photo- All } \\
\text { streets documentations }\end{array}$ & & Figure 4 \\
\hline
\end{tabular}




\begin{tabular}{|l|l|l|l|l|}
\hline & & $\begin{array}{l}\text { Existing/ Standards } \\
\text { Streets sections }\end{array}$ & $\begin{array}{l}\text { Buildings' entrances and streets, } \\
\text { Sidewalks network- sections "campus } \\
\text { streets dimensions" }\end{array}$ & Figure 5 \\
\hline $\begin{array}{l}\text { Analytical } \\
\text { calculation }\end{array}$ & Microsoft excel & Relation graphs & & Figure 9 \\
\hline \multicolumn{2}{|l|}{ Source of all data either prepared by research or extracted by the software's mentioned } \\
\hline
\end{tabular}

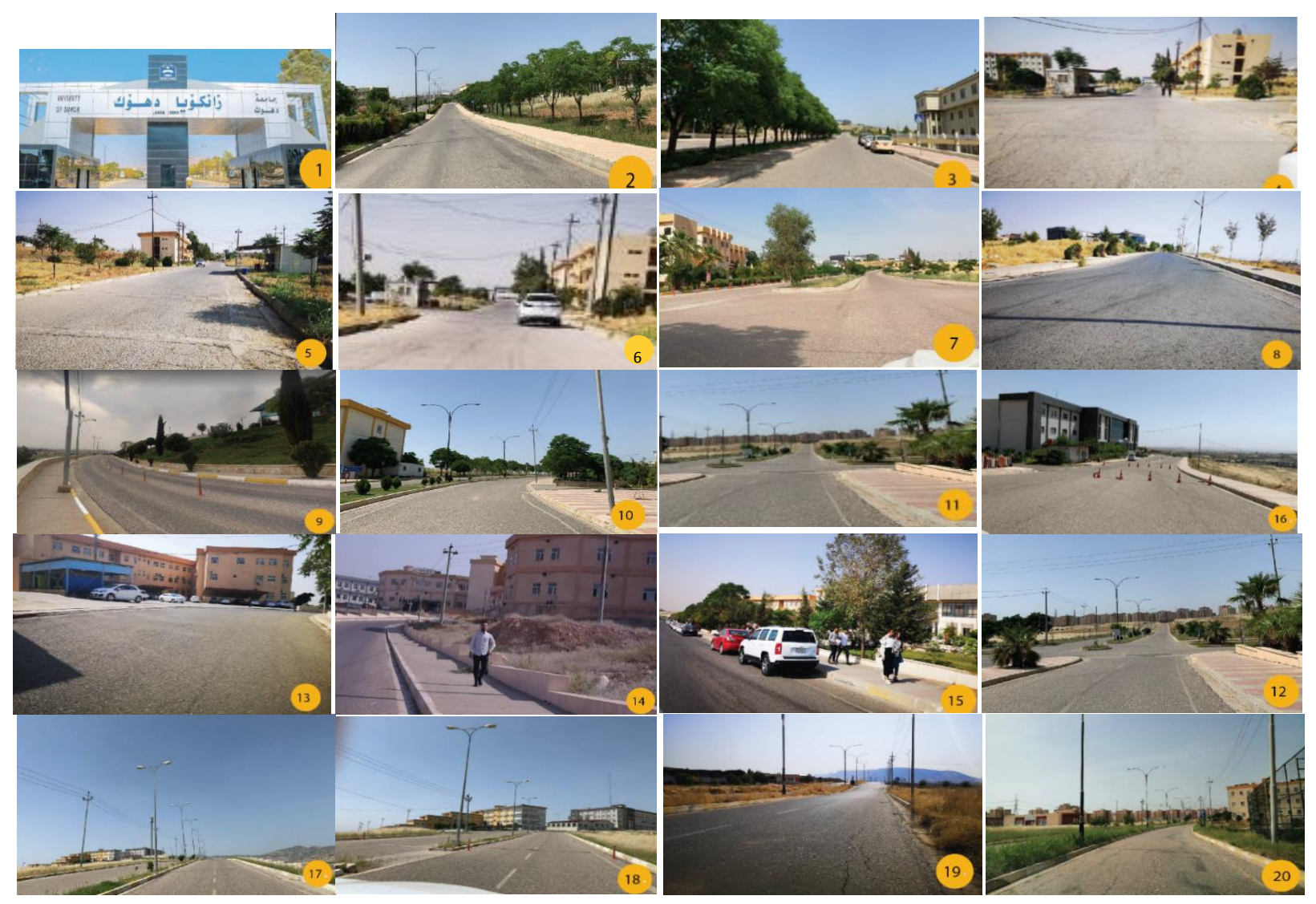

Figure 3 Visual documentation for the investigated locations (Observation points 1-20)

\section{SPACE SYNTAX ANALYSIS}

Hillier and his colleagues addressed the physical characteristics of street networks, as an influencing factor that affects an individual's choice of a street segment (Hillier, Penn, Hanson, Grajewski, \& Xu, 1993). In the UoD campus, there is no designed pathways (Table 3), That is discouraged students and staff to walk to their destination Figure 1. Where the researchers observed that the lack of proper connection in pedestrian paths in some streets and sidewalks has been considered as a major obstacle for walkability. The research interpretation was done based 
on a space syntax analysis has been carried out using depthmapX Table 4. As below:(1) Connectivity "measures the number of immediate neighbors that are directly connected to space" (2) Integration "It describes the average depth of space to all other spaces in the system". Local Integration is considered as immediate affordable accessibility through the network, where a trip of 3 steps is considered. (3) Global choice is a "dynamic global measure of the "flow" through space. Where Space has a strong choice-value when many of the shortest paths, connecting all spaces to all spaces of a system, passes through it". (Klarqvist,1993) (Klarqvist, 2015). For the University of Duhok, the syntactical properties have been measured and the result in Figure 7.a., b., C., to imagine UoD connectivity (Connectivity Index), Figure 7.a. showing how well the UoD network is connected. The average degree of street connectivity inside the campus, it shows the extent of the alterations from one line to another and the availability of sidewalk Figure 9. a. depthmapX shows the Minimum value of Connectivity is equal to (1.00) and the Maximum, is (10.00) Table 4. Figure 7.b. shows the average degree of Integrated streets for the campus for Local Integration analyses, where it showed that the highest values for depthmapX is (2.52), and the lowest values (0.33) Table 4. Global integration, Figure 7.c analyses showed that the highest values for depthmapX are (1.9) and the lowest values (0.37), which considered slightly low recall Table 4. Comparing this value with the relationship with $\%$ of a street with sidewalk Figure 9.c.

Table 3 UoD sidewalk standards compare to local and international standards

\begin{tabular}{|c|c|c|c|c|c|c|}
\hline & \multicolumn{4}{|c|}{ UoD street } & \multicolumn{2}{|c|}{ Walk side standards } \\
\hline & & \multicolumn{2}{|c|}{ UoD Walk side } & & & \\
\hline & & Available & Width & & & \\
\hline Fig 3.1. & 7 & Yes & 4 & Fig 5.a. & 1.5 & \\
\hline Fig 3.9. & 7 & Yes & 5 & Fig 5.b. & 1.5 & \\
\hline Fig 3.20 . & 6 & No & - & Fig 5.c. & - & \\
\hline Fig 3.15 & 6 & Yes & 5.6 & Fig 5.d. & No standards & \\
\hline Fig 3.19 & 7 & No & - & Fig 5.e. & 1.5 & \\
\hline Fig 4.9. & 6 & Yes & 8 & Fig 5.f. & No standards & \\
\hline Fig 4.10. & 6 & Yes & 8 & Fig 5.g. & No standards & \\
\hline \multicolumn{7}{|c|}{$\begin{array}{l}\text { a) According to Duhok municipality developmentstandards, } 1.5 \mathrm{~m} \text { for } 6.1 \mathrm{~m} \text { street width (Abdulsamadet al., 2019) b) (San Francisco, } \\
\text { 2020) see Figurec) (San Diego, 2017) }\end{array}$} \\
\hline
\end{tabular}




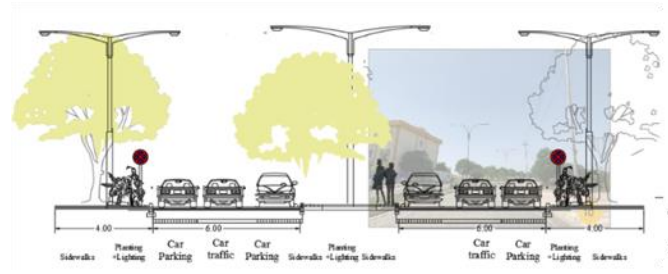

(g)

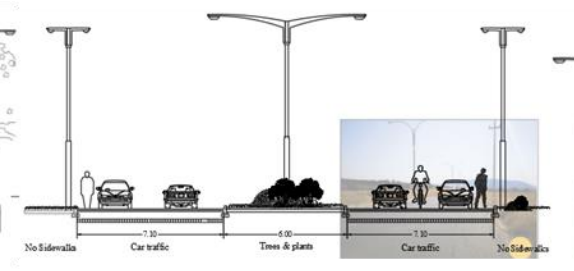

(e)

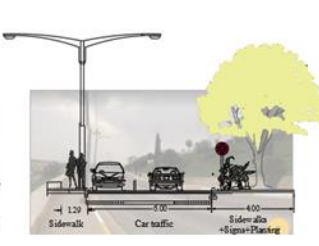

(f)

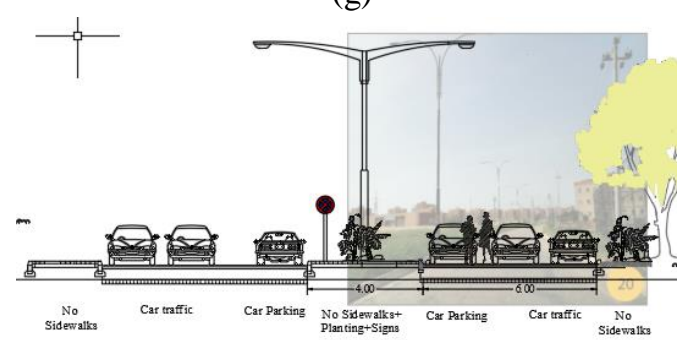

(c)

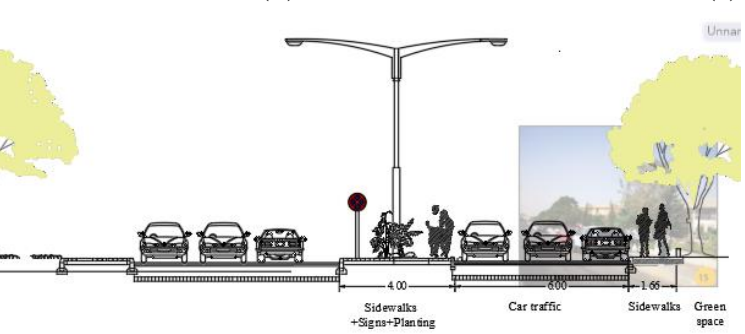

(d)

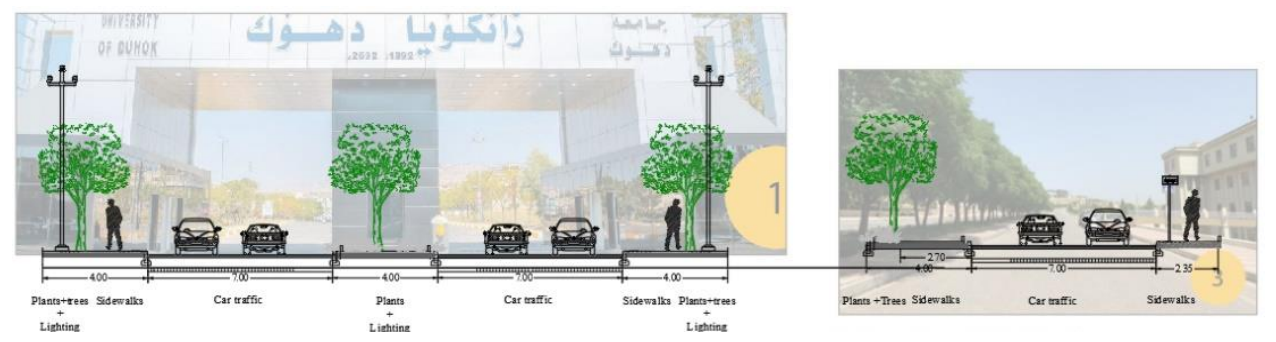

(a)

(b)

Figure 4 Campus streets Graphical analysis (Section) for a sample of selected observation locations, from obserbvation points (a) No.1,(b) No. 3, (c) No.20, (d) No. 15, (e) No.19, (g) No.10, (f) No.9

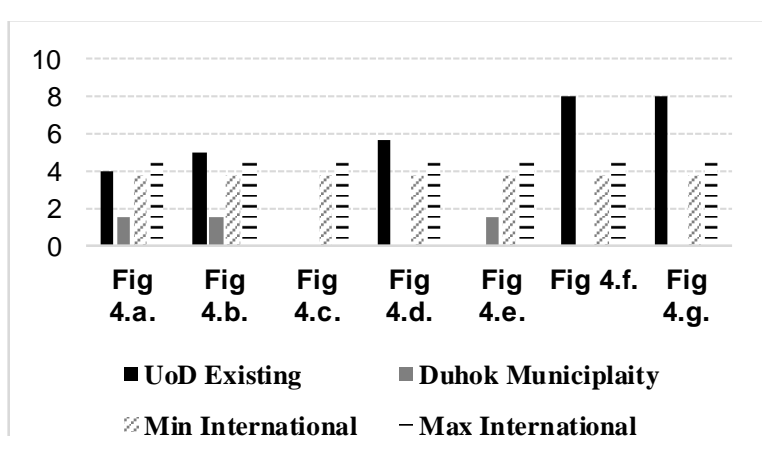

(a)

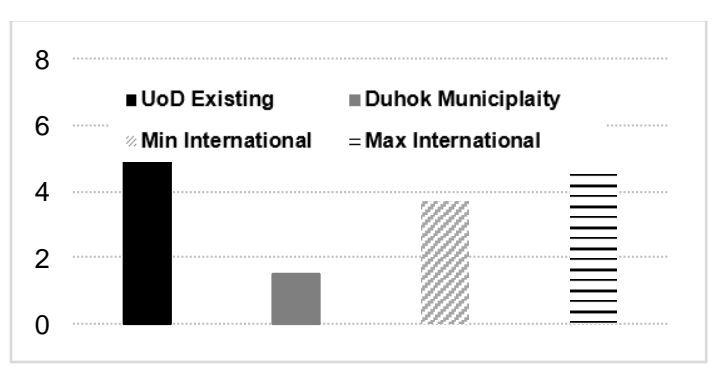

(b)

Figure 6 sidewalk standard comparison, (a) Dimensions of UOD sidewalks, Duhok Municipality and Intentional standard, (b) Mean measurement for UOD sidewalks, Duhok municipality and international standards. 
Table 4: UoD Campus: Space Syntax Parameters

\begin{tabular}{|l|l|l|}
\hline Parameter & Value & depthmapX \\
\hline \multirow{7}{*}{} & Minimum & 1 \\
\cline { 2 - 3 } & Maximum & 10 \\
\cline { 2 - 3 } & Mean & 3.39 \\
\cline { 2 - 3 } & Standard Deviation & 2.02 \\
\hline \multirow{7}{*}{} & Minimum & 0.33 \\
\cline { 2 - 3 } & Maximum & 2.56 \\
\cline { 2 - 3 } & Mean & 1.44 \\
\cline { 2 - 3 } & Standard Deviation & 0.50 \\
\hline \multirow{7}{*}{} & Minimum & 0.37 \\
\cline { 2 - 3 } & Maximum & 1.90 \\
\cline { 2 - 3 } & Mean & 0.73 \\
\cline { 2 - 3 } & Standard Deviation & 0.25 \\
\hline
\end{tabular}

\begin{tabular}{|l|l|l|}
\hline Parameter & Value & depthmapX \\
\hline \multirow{5}{*}{} & Minimum & 0 \\
\cline { 2 - 3 } & Maximum & 184 \\
\cline { 2 - 3 } & Mean & 20.89 \\
\cline { 2 - 3 } & Standard Deviation & 28.40 \\
\hline \multirow{5}{*}{} & Minimum & 0 \\
\cline { 2 - 3 } & Maximum & 7580 \\
\cline { 2 - 3 } & Mean & 1119.51 \\
\cline { 2 - 3 } & Standard Deviation & 1494.49 \\
\hline
\end{tabular}

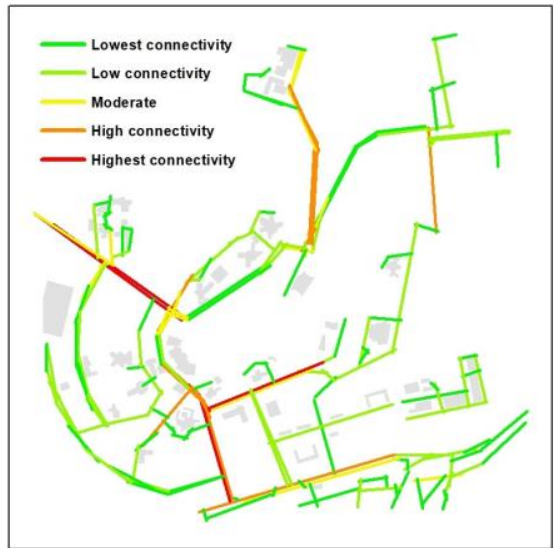

(a)

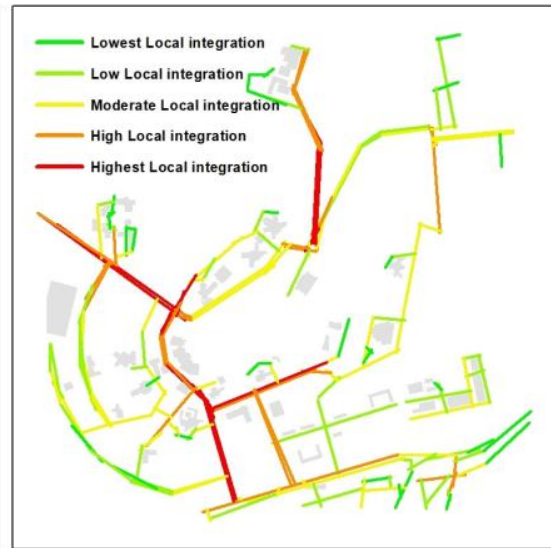

(b)

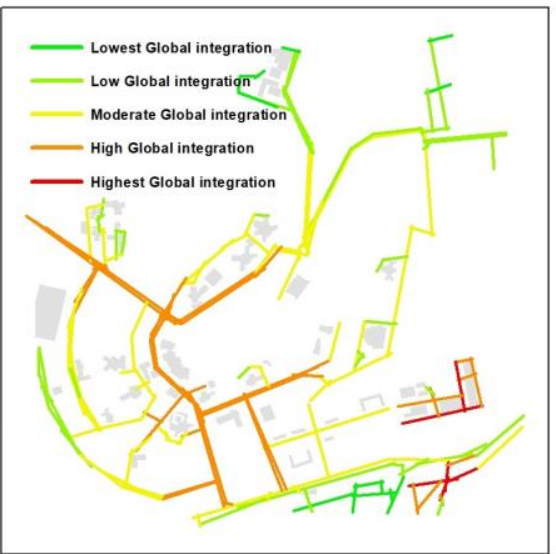

(c)

Figure 7 UoD Space syntax-depthmapX

(a) Connectivity Index, (b) Local integration Index, (c) Global integration Index

(b)

Finally, Global Choice in Figure 8.a.,8. b., the highest values (18300) and the lowest equal to (0), while the local measure for the same variable, the highest (572) and the lowest value (0) and the average for both of them respectively (2099.4) (40.36). Table 4 . The graph in Figure 8 shows the Local and Global choice index comparison. Statically, Figure (9) showing that there is an effect of the availability of street sidewalk infrastructure on campus connectivity, integration, and individuals' choice. Where Global Choice is the most effective index compared to (Connectivity and integration indexes) are negatively stepping down as well, but the choice is the most interrelated index. 


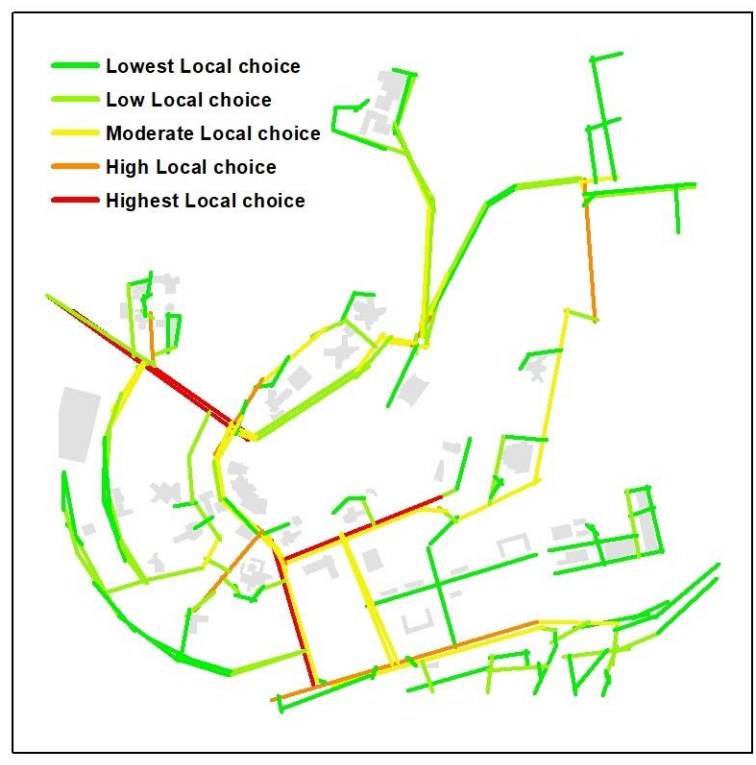

(a)

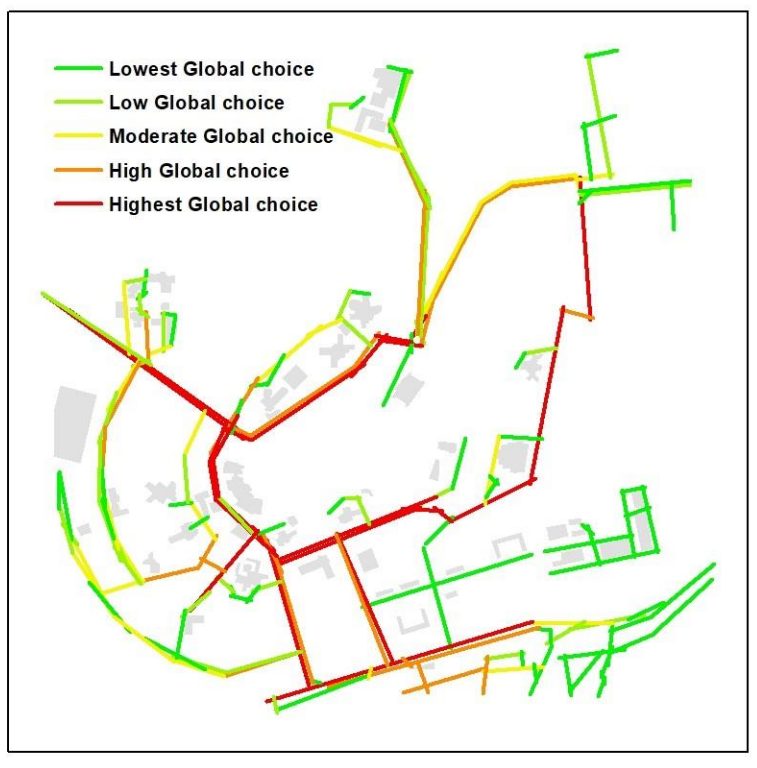

(b)

Figure 8 UoD Space syntax-depthmapX

(a) Local choice Index, (b) Global choice Index

To show the relationship between the availability of sidewalks and Space syntax analysis, a statistical analysis has been done for (1) calculating the percentage of "with sidewalk infrastructure", (2) the percentage of "without sidewalk infrastructure" per Space syntax category Table $(5,6)$ according to the given formula:

$$
\begin{aligned}
& =\left(\frac{A}{C}\right) * 100 \\
& =\left(\frac{B}{C}\right) * 100
\end{aligned}
$$

Where: $A=$ total length of lines segment per space syntax index (Connectivity, Integration, Choice) categorize (low, medium, high) with sidewalk infrastructure, $\mathrm{B}=$ total length of lines segment for certain space syntax index categorizes (low, medium, high) without sidewalk infrastructure, $\mathrm{C}=$ total length of lines segment for certain space syntax index categorizes (low, medium, high) all in metric units. 
Table 5 Comparison between the effect of a line segment with sidewalk infrastructure and without sidewalk infrastructure on the readings of Space syntax indicators in depthmapX

\begin{tabular}{|l|l|c|c|}
\hline \multirow{2}{*}{ Indicator } & \multicolumn{2}{|c|}{ depthmapX } \\
\cline { 3 - 4 } \multicolumn{1}{|c|}{} & \% of line segments with walk side & $\begin{array}{l}\text { \% of line segments without walk } \\
\text { side }\end{array}$ \\
\hline \multirow{3}{*}{} & Lowest connectivity & 11 & 89 \\
\cline { 2 - 4 } & Medium connectivity & 23 & 77 \\
\cline { 2 - 4 } & Highest connectivity & 30 & 70 \\
\hline \multirow{3}{*}{} & Lowest Local Integration & 5 & 65 \\
\cline { 2 - 4 } & Medium Local Integration & 33 & 60 \\
\cline { 2 - 4 } & Highest Local Integration & 40 & 100 \\
\hline & Lowest Global Integration & 0 & 77 \\
\cline { 2 - 4 } & Medium Global Integration & 23 & 100 \\
\cline { 2 - 4 } & Highest Global Integration & 0 & \\
\hline
\end{tabular}

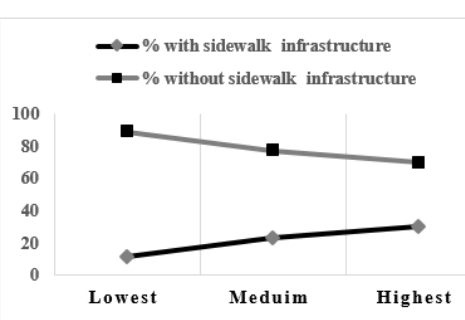

(a)

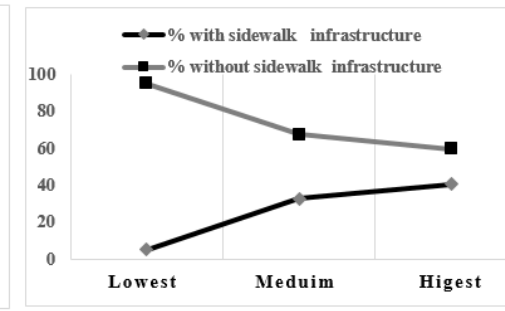

(b)

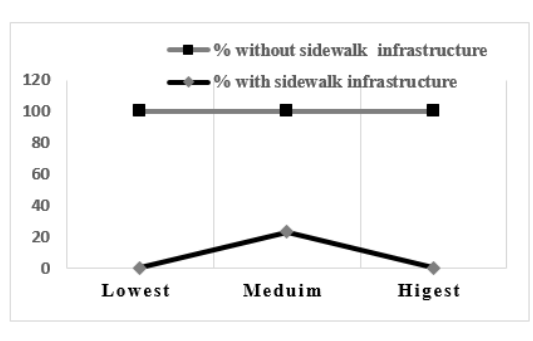

(c)

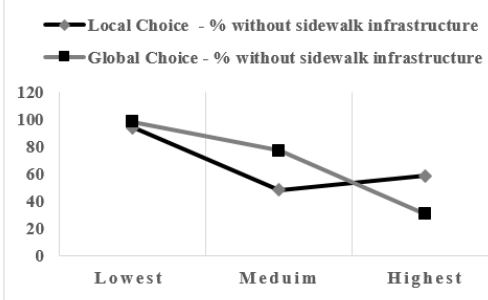

(d)

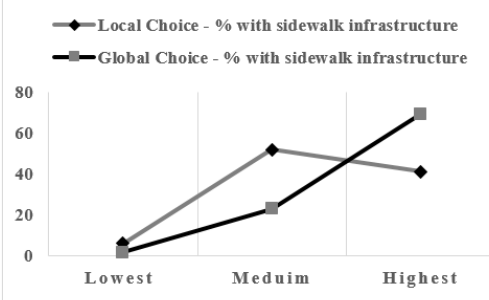

(e)

Figure 9 Statistical comparison for percentage of sidewalk infrastructure availability and its effect on space syntax index, (a) Connectivity Index, (b) Local integration, (c) Global integration (d) Choice to without infrastructure walk sides (e) Choice to with infrastructure walk sides

Table 6 Comparison between the effect of a line segment with sidewalk infrastructure and without sidewalk infrastructure on the readings of Space syntax indicators in depthmapX

\begin{tabular}{|c|c|c|c|c|}
\hline & $\begin{array}{c}\text { Local Choice with \% of } \\
\text { with sidewalk }\end{array}$ & $\begin{array}{c}\text { Global Choice with \% of } \\
\text { with sidewalk }\end{array}$ & $\begin{array}{c}\text { Local Choice with \% of } \\
\text { without sidewalk }\end{array}$ & $\begin{array}{c}\text { Global Choice with \% of } \\
\text { without sidewalk }\end{array}$ \\
\hline lowest & 6 & 2 & 94 & 98 \\
\hline Medium & 52 & 23 & 48 & 77 \\
\hline highest & 41 & 69 & 59 & 31 \\
\hline
\end{tabular}




\section{CONCLUSION AND RECOMMENDATION}

The conclusion and recommendation for this study are: University of Duhok Campus need to considered and implemented an alternative "transportation mode network" such as (public transport, Bike, standardized sidewalk lanes). Where the analysis is showing the main obstacles for pedestrian can be the lack of proper connection and walk sides in some streets and walking paths. Furthermore, the quality of the pavements, study of shadow and shade and street furnishings had an effect as well. Similarly, establishing an organizing traffic system (e.g. traffic lights at intersections) is highly advised for pedestrian safety and timesaving. In addition, maintenance and reconstruction of sidewalks, adding Crosswalk lines on intersections is very essential. Finally, a more researches are required for universities friendly campus and walkable environments. An interview with student and staff is necessary for the matter of comprehensive research. An awareness campaign about the mental and psychological advantage of walking and cycling "Healthy mind in a healthy body".

\section{REFERENCES}

Abdulsamad, Q., Mohammed, J., \& Abdullah, P. 2019. Evaluation of the existing sidewalks In Duhok City. Journal of Duhok University, 22(1), 58-74.

Bolshakov, A. 2019. Urban topology of university campus. IOP Conference Series: Materials Science and Engineering, 667, 012014.

Brusaporci, S. 2015. The representation of architectural heritage in the digital age. In Encyclopedia of Information Science and Technology, Third Edition, 4195-4205: IGI Global.

Brusaporci, S. 2018. Graphical Analysis 2.0: Digital Representation for Understanding and

Fruin, J. 1992. Designing for pedestrians. Public Transportation United States.

Hillier, B., Hanson, J., \& Peponis, J. 1984. What do we mean by building function? 
Hillier, B., \& Iida, S. 2005. Network effects and psychological effects: a theory of urban movement. Paper presented at the Proceedings of the 5th international symposium on space syntax.

Hillier, B., Penn, A., Hanson, J., Grajewski, T., \& Xu, J. 1993. Natural movement: or, configuration and attraction in urban pedestrian movement. Environment and Planning B: Planning and design, 20(1), 29-66.

Jones, P., Marshall, S., \& Boujenko, N. 2008. Creating more people-friendly urban streets through 'link and place'street planning and design. IATSS research, 32(1), 14-25.

Keat, L., Yaacob, N., \& Hashim, N. 2016. Campus walkability in Malaysian public universities: A case-study of Universiti Malaya. PLANNING MALAYSIA, 14(5).

Klarqvist, B. 2015. A space syntax glossary. NA, 6(2).

San Diego, G. 2017. Street Design Manual

San Francisco, G. 2020. A guide to making better streets plan in San Francisco,Design guide.

Southworth, M. 2005. Designing the walkable city. Journal of Urban Planning and Development, 131(4), 246-257.

Turner, A. 2004. Depthmap 4: a researcher's handbook.

Van Nes, A. 2014. Space syntax in theory and practice. In Geodesign by Integrating Design and Geospatial Sciences (pp. 237-257): Springe 